TI 2000-40/2

Tinbergen Institute Discussion Paper

\title{
Comprehensive Definitions of Breakdown-Points for Independent and Dependent Observations
}

Marc G. Genton

André Lucas 
Tinbergen Institute

The Tinbergen Institute is the institute for economic research of the Erasmus Universiteit Rotterdam, Universiteit van Amsterdam and

Vrije Universiteit Amsterdam.

Tinbergen I nstitute Amsterdam

Keizersgracht 482

1017 EG Amsterdam

The Netherlands

Tel.: +31.(0)20.5513500

Fax: $\quad+31 .(0) 20.5513555$

Tinbergen I nstitute Rotterdam

Burg. Oudlaan 50

3062 PA Rotterdam

The Netherlands

Tel.: $\quad+31 .(0) 10.4088900$

Fax: $\quad+31 .(0) 10.4089031$

Most TI discussion papers can be downloaded at

http://www.tinbergen.nl 


\title{
Comprehensive Definitions of Breakdown-Points for Independent and Dependent Observations
}

\author{
Marc G. Genton and André Lucas*
}

May 3, 2000

\begin{abstract}
We provide a new definition of breakdown in finite samples with an extension to asymptotic breakdown. Previous definitions center around defining a critical region for either the parameter or the objective function. If for a particular outlier constellation the critical region is entered, breakdown is said to occur. In contrast to the traditional approach, we leave the definition of the critical region implicit. Our definition encompasses all previous definitions of breakdown in both linear and non-linear regression settings. In some cases, it leads to a different notion of breakdown than other procedures available. An advantage is that our new definition also applies to models for dependent observations (time-series, spatial statistics) where current breakdown definitions typically fail. We illustrate our points using examples from linear and non-linear regression as well as time-series and spatial statistics.
\end{abstract}

Key words: Bias curve; Linear regression; Non-linear regression; Outliers; Spatial statistics; Statistical robustness; Time series.

${ }^{*}$ Marc G. Genton is Lecturer, Department of Mathematics, 2-390, Massachusetts Institute of Technology, 77 Massachusetts Avenue, Cambridge, MA 02139-4307, genton@math.mit.edu. André Lucas is Associate-Professor, Department of Finance, ECO/FIN, Vrije Universiteit, De Boelelaan 1105, 1081HV Amsterdam, the Netherlands, alucas@econ.vu.nl. André Lucas thanks the Dutch Organization for Scientific Research (N.W.O.) for financial support. 


\section{Introduction}

The issue of qualitative robustness and especially the definition of breakdown has made considerable progress over the last three decades. Hampel (1971) defined breakdown as the fraction of contamination (or outliers) that suffices to drive the estimator beyond all bounds. Since the original introduction of the concepts of breakdown and the breakdown-point by Hampel (1971), the breakdown-point has been extended to finite samples (Donoho and $\mathrm{Hu}-$ ber, 1983), bounded parameter spaces, dependent observations (Martin and De Jong, 1977; Martin, 1980), test statistics (He et al., 1990; He, 1991), and non-linear regression models (Stromberg and Ruppert, 1992; Sakata and White, 1995, 1998). Especially Stromberg and Ruppert (1992) and Sakata and White (1995) convincingly argue that the bias in the parameter estimates is not always a good criterion to assess breakdown of an estimator. Instead, Stromberg and Ruppert propose to consider the fraction of contamination that drives at least one of the fitted values to its supremum or infimum. Sakata and White argue that the fitted value may sometimes not be a satisfactory criterion either, and therefore propose several alternative criterion functions to assess breakdown.

Though these alternative definitions cover a wide range of models and estimators, one can easily construct examples that are not covered by the available definitions. A very simple example is given by the autoregressive time-series model of order 1 ,

$$
Y_{i}=\phi Y_{i-1}+e_{i}
$$

with $\phi \in(-1,1)$ and $e_{i}$ an i.i.d. innovation. Suppose $Y_{i}$ is observed with error as $\tilde{Y}_{i}=Y_{i}+Z_{i}$, where $Z_{i}=\zeta$ when $i=i_{0}$ for a single $i_{0} \in\{1, \ldots, n-1\}$, and $Z_{i}=0$ otherwise. Then the OLS estimator of $\phi$ based on the contaminated sample $\tilde{Y}_{1}, \ldots, \tilde{Y}_{n}$, is given by

$$
\hat{\phi}=\frac{\sum_{i=2}^{n} \tilde{Y}_{i} \tilde{Y}_{i-1}}{\sum_{i=2}^{n} \tilde{Y}_{i-1}^{2}}=\frac{\zeta \cdot\left(Y_{i_{0}-1}+Y_{i_{0}+1}\right)+\sum_{i=2}^{n} Y_{i} Y_{i-1}}{\zeta^{2}+2 \zeta Y_{i_{0}}+\sum_{i=2}^{n} Y_{i-1}^{2}} .
$$

Clearly, as $\zeta \rightarrow \infty, \hat{\phi} \rightarrow 0$. So the OLS estimator in this simple time-series model breaks with one outlier to zero, which is at the center of the parameter space. This form of breakdown typically rules out the classical definition of Hampel, because the estimator does not diverge. Moreover, it also violates the straightforward extension of Hampel's definition to compact parameter spaces. In that definition, breakdown occurs if the estimator is pushed to the edge of the parameter space. Here, however, the estimator does not go to the edge, but rather to the center of the parameter space. Also note that this 
simple example does not fit the more recent definitions of breakdown either. In particular, following the definition of He and Simpson (1992, 1993), breakdown occurs if the supremum bias is reached. This, however, need not be the case if $\phi$ is negative or positive, in which case the sup bias is reached upon breakdown to plus one or minus one instead of zero, respectively. Alternatively, Stromberg and Ruppert and also Sakata and White define breakdown as the point where the model's fit $\left(\hat{\phi} Y_{i-1}\right)$ or some other criterion function tends to either its supremum or its infimum for some observation in the sample. Clearly, this would again induce breakdown to either plus or minus one given the restricted parameter space, and not breakdown to zero.

Given the drawbacks of the previous definitions available, we introduce a new concept of breakdown. All previous definitions make explicit use of a criterion function combined with a critical region. For example, Hampel's original definition uses the absolute bias as the criterion function and infinity as the critical region. If the criterion function enters the critical region for a certain fraction of outliers/contamination, breakdown is said to have occurred. Following Sakata and White (1995), we consider a specific model badness measure as our criterion function. This encompasses the definitions of Hampel (badness is bias) as well as Stromberg and Ruppert (badness is model fit). In contrast to previous work, however, we leave the definition of the critical region implicit. In particular, we look for the fraction of contamination such that the set of possible badness values under extreme outlier configurations does not expand any more if additional outliers are added. In this way, we are able to accomodate most of the earlier definitions of breakdown. In addition, we also cover situations of breakdown that are not covered by the earlier definitions. We illustrate the main issues with examples from linear and non-linear regression as well as time-series and spatial statistics.

In some cases, our definition of breakdown gives a different breakdown point than available definitions. We provide a typical example in the nonlinear regression context, confronting our breakdown point with that of Stromberg and Ruppert. The new notion of breakdown checks whether the non-contaminated sample information still has some influence on the estimator. If this is no longer the case, the estimator is said to have broken down. This may happen even in case the model's fit over a pre-specified domain of interest remains bounded.

The remainder of the paper is set up as follows. In Section 2 we introduce the basic notation and our new definition of breakdown for finite samples. The definition is related to alternative ones in Section 3. Some illustrative examples are given in Section 4. Section 5 extends the definition of the breakdown-point to the asymptotic case and provides some illustrations. Section 6 concludes. 


\section{Definition of Breakdown}

We consider a sample of size $n$,

$$
\bar{Y}_{n}=\left(Y_{1}, \ldots, Y_{n}\right)
$$

The sample may consist of i.i.d. observations as well as dependent observations, e.g., a time-series. The estimator of the $K$-dimensional parameter vector $\theta$ is defined as a function of the sample, i.e.,

$$
\hat{\theta}=\hat{\theta}\left(\bar{Y}_{n}\right)
$$

We introduce outliers through a contaminating sample $\bar{Z}_{n, m}^{\zeta}$. Here $n$ denotes the sample size again, $m$ represents the number of outliers in the contaminating sample, and $\zeta$ indicates the magnitude of the outliers. For example, in the context of estimating the location of an i.i.d. sample, $\bar{Z}_{n, m}^{\zeta}$ typically contains $(n-m)$ zeros and $m$ non-zeros. Using the concept of a contaminating sample rather than individual outliers allows us to consider outlier patterns that exhibit more structure than in the regression setting. For example, in a timeseries setting we distinguish between additive outliers (AOs) or replacement outliers (ROs), and innovation outliers (IOs). The former can be considered as pure measurement errors, whereas the latter are exceptional shocks that satisfy the feed-through mechanism of the time-series process. Consider a simple autoregression as in (1). An additive or replacement outlier can then be studied by specifying

$$
\bar{Z}_{n, 1}^{\zeta}=(0, \ldots, 0, \zeta, 0, \ldots, 0)
$$

for some $\zeta \in \mathbb{R}$, while an innovation outlier corresponds to

$$
\bar{Z}_{n, 1}^{\zeta \prime}=\left(0, \ldots, 0, \zeta, \phi \zeta, \phi^{2} \zeta, \ldots\right)
$$

Similarly, in the context of spatial statistics, consider a simple simultaneous autoregressive model of order 1 ,

$$
Y_{i}=\phi\left(Y_{i-1}+Y_{i+1}\right)+e_{i}
$$

Again, an additive or replacement outlier can be studied by specifying

$$
\bar{Z}_{n, 1}^{\zeta}=(0, \ldots, 0, \zeta, 0, \ldots, 0)
$$

for some $\zeta \in \mathbb{R}$, while an innovation outlier corresponds to

$$
\bar{Z}_{n, 1}^{\zeta \prime}=\left(\ldots, \phi^{2} \zeta, \phi \zeta, \zeta, \phi \zeta, \phi^{2} \zeta, \ldots\right)
$$


We define $\mathcal{Z}_{n, m}^{\zeta}$ as the set of allowable outlier constellations. For example, the set of allowable IOs typically includes $\bar{Z}_{n, 1}^{\zeta \prime}$, but not $\bar{Z}_{n, 1}^{\zeta}$ as defined above.

As mentioned in the introduction, defining breakdown in terms of bias in the estimator is not always satisfactory. Especially if the model is nonlinear, criteria other than parameter stability may be more relevant. See also the arguments raised in Stromberg and Ruppert (1992) and Sakata and White (1995). Following Sakata and White (1995), therefore, we introduce a badness measure

$$
R_{n}\left(\theta, \bar{Y}_{n}\right) \in \mathbb{R}^{+} .
$$

Sakata and White define breakdown as the fraction of contamination that drives the badness measure to its supremum. As the badness measure may coincide with the bias, this encompasses the Donoho-Huber definition of breakdown. The main disadvantage of Sakata and White's definition is that estimators may already have been broken before the badness measure reaches its supremum. A nice example was given in the introduction for the OLS estimator of the $\operatorname{AR}(1)$ parameter, where badness is taken as bias. The failure of the Sakata and White definition is due to the fact that the breakdown region is made explicit, in particular, the supremum badness. In our definition, we leave the breakdown region implicit. First, we introduce the boundary set of possible badness outcomes for contaminated samples,

$$
\bar{R}_{n}\left(\bar{Y}_{n}, \mathcal{Z}_{n, m}^{\zeta}\right)=\partial\left(\bigcup_{\bar{Z}_{n, m}^{\zeta} \in \mathcal{Z}_{n, m}^{\zeta}} R_{n}\left(\hat{\theta}\left(\bar{Y}_{n}+\bar{Z}_{n, m}^{\zeta}\right), \bar{Y}_{n}\right)\right),
$$

where $\partial(A)$ is the boundary of the set $A$. We allow for $\bar{R}_{n}(\cdot)$ to contain $\pm \infty$. Second, we introduce the perturbed uncontaminated sample $\bar{Y}_{n}^{\delta}$, with $\max _{i}\left|Y_{i}^{\delta}-Y_{i}\right|<\delta$. The perturbed sample is needed in case there are duplicate observations in the sample. We now introduce the following definition of breakdown.

Definition 1 The breakdown-point of the estimator $\hat{\theta}$ of $\theta$ is given by

$$
\begin{array}{r}
\varepsilon \equiv \lim _{\delta \rightarrow 0} \min \left\{\frac{m-1}{n} \mid \lim _{\zeta \rightarrow \infty} \bar{R}_{n}\left(\bar{Y}_{n}^{\delta}, \mathcal{Z}_{n, m}^{\zeta}\right) \bigcap\right. \\
\left.\lim _{\zeta \rightarrow \infty} \bar{R}_{n}\left(\bar{Y}_{n}^{\delta}, \mathcal{Z}_{n, m+1}^{\zeta}\right) \neq \emptyset \quad \forall \bar{Y}_{n}^{\delta}\right\} .
\end{array}
$$

The definition looks for the smallest fraction of extreme outliers for which the boundary of the set of possible badness values does not expand any more 
in all directions if an additional outlier is added to the sample. Consider for example the mean as an estimator of location. By adding either one or two extreme outliers, one can drive the estimator to $+\infty$. This implies that the boundary set contains $+\infty$ for both $m=1$ and $m=2$. Therefore, following the definition, the breakdown point of the mean is 0 . It is important to note that the definition is implemented for extreme outliers. We are not concerned here with the possibly huge biases in the estimator for less extreme outliers. Note, however, that $\zeta \rightarrow \infty$ need not imply that the outliers tend to (plus or minus) infinity. For example, in the context of scale estimation it can be worthwhile to consider outliers for which the nonzero elements of $\bar{Z}_{n, m}^{\zeta}$ are either $\zeta$ or $\zeta^{-1}-Y_{i}$. These alternative types of outliers can be used to check for explosion or implosion of the scale estimator, respectively. The restriction to extreme outliers rather than arbitrary outlier configurations makes our definition easier to apply. Moreover, it still includes most situations of practical interest.

It is good to note here that the boundary set of possible badness outcomes naturally contains its supremum, that is the supremum over extreme outlier configurations. Using our definition of breakdown, this means that if the sup-badness curve is flat, the estimator is broken. Note that the sup-badness curve may increase further when even more outliers are added, meaning that the estimator may not have been broken in the sense of Stromberg and Ruppert or Sakata and White. A nice example in the time-series setting is provided in Section 5 .

The sample perturbation $\bar{Y}_{n}^{\delta}$, as mentioned earlier, is needed if there are duplicate entries in the sample. Consider for example the median of the sample

$$
1,3,3,3,3,3,7 \text {. }
$$

Consider the case where badness is the value of the estimator. The median of this sample is 3 . If one or two of the observations are changed, the median remains 3 , and therefore the boundary set of possible badness values does not expand. Clearly, however, the estimator has not been broken. If the sample is slightly perturbed, e.g., by adding $i \cdot 10^{-6}$ to the $i$ th observation, the median changes if either one or two observations are altered. Therefore, the boundary set expands from $\left\{3+4 \cdot 10^{-6}\right\}$ to $\left\{3+2 \cdot 10^{-6}, 3+6 \cdot 10^{-6}\right\}$ for the perturbed sample and the estimator has not been broken.

It is worthwhile to mention several possible modifications and/or extensions to our definition. First, in some cases it is informative to look for the point where the boundary set does not expand for the next $k$ (extreme) 
outliers instead of the next outlier only,

$$
\begin{aligned}
& \varepsilon^{k} \equiv \lim _{\delta \rightarrow 0} \min \left\{\frac{m-1}{n} \mid \lim _{\zeta \rightarrow \infty} \bar{R}_{n}\left(\bar{Y}_{n}^{\delta}, \mathcal{Z}_{n, m}^{\zeta}\right) \bigcap\right. \\
& \left.\lim _{\zeta \rightarrow \infty} \bar{R}_{n}\left(\bar{Y}_{n}^{\delta}, \mathcal{Z}_{n, m+\ell}^{\zeta}\right) \neq \emptyset \quad \forall \bar{Y}_{n}^{\delta}, \quad \text { for } \ell=1, \ldots, k\right\} .
\end{aligned}
$$

In particular, by letting $k$ tend to $n$, we look for the fraction of outliers that makes the boundary set constant in at least one direction. A second point concerns the use of the uncontaminated $\left(\bar{Y}_{n}\right)$ or the contaminated $\left(\bar{Y}_{n}+\bar{Z}_{n, m}^{\zeta}\right)$ sample as the second argument in the badness function $R_{n}(\hat{\theta}, \cdot)$ in $(6)$. In our definition, we follow Sakata and White (1995) and use the uncontaminated sample. In some cases, however, it can also be useful to insert the contaminated sample into the badness function, see for example Genton (1998b), and Ma and Genton (2000). A third possible extension concerns the direction of breakdown. So far, we concentrate on there being some direction in which the boundary badness set does not expand. In certain cases, however, it might be interesting to obtain more information on the direction in which the estimator is most likely to break down. For example, in the scale estimation problem discussed earlier, we might distinguish between implosion and explosion of the scale estimator, depending on whether the lower or the upper endpoint of the boundary set remains fixed.

In the next section, we discuss the relation between our definition and the definitions available in the literature. In Section 4 we give some illustrative examples, further highlighting the difference of our definition with alternative definitions of breakdown.

\section{Relation to Available Definitions}

Consider the i.i.d. regression model

$$
Y_{i}=X_{i} \beta+e_{i}
$$

with $\bar{Y}_{n}=\left(\left(Y_{1}, X_{1}\right), \ldots,\left(Y_{n}, X_{n}\right)\right)$. Any reasonable estimator for $\beta$ will change with the value of $\bar{Y}_{n}$. Therefore, if we take the badness function to be the bias, the only way to get a constant boundary set is to let the estimator diverge to plus or minus infinity. This reproduces the standard definition of breakdown by Donoho and Huber. In other cases, for example dependent observations, the Donoho-Huber breakdown point will provide an upper bound for the breakdown point from our definition, given that the badness function is the (absolute) bias. 
Stromberg and Ruppert (1992) define breakdown in terms of model fit. If we take model fit as our badness measure, we again obtain that the Stromberg-Ruppert breakdown point provides an upper bound for our new definition of the breakdown point. That the upper bound may be strict is illustrated in the next section using the non-linear Michaelis-Menten regression model. In our definition, an estimator has broken if the remaining uncontaminated observations have no effect on the estimator any more. This may happen even if the model's fit is still below its supremum or above its infimum. In our view, it is more natural to say that the estimator has been broken if its value is totally dictated by the outliers in the sample, while the uncontaminated observations do not have an effect. As the definition of Sakata and White (1995) is very similar to that of Stromberg and Ruppert, similar comments apply to it. Also note that both definitions fail to accommodate breakdown in the simple autoregressive model (1).

Martin and De Jong (1977) and Martin (1980) define breakdown for the autoregressive time-series setting. In particular, they consider breakdown towards zero and to plus or minus one as the relevant notions of breakdown. Though we completely agree with their analysis, their approach is rather focussed on the time-series setting and only discusses asymptotic robustness. Our definition, by contrast, quite naturally accommodates both the regression setting and the time-series setting, see the examples in the next sections. Moreover, we also provide a definition of breakdown that can be used in finite samples.

Other previous definitions of breakdown points for dependent observations have been studied by Genton (1998b) for spatial statistics and Ma and Genton (2000) in the context of time series. Both approaches are extending the traditional breakdown point to a spatial and temporal one for variogram and autocovariance estimators respectively. The reason is that these estimators are based on differences between observations apart by a (lag) vector $h$ and usually have a known breakdown-point with respect to these differences. However, practitioners are interested in the breakdown point with respect to the initial observations that are located in space or time. Therefore, one has to study the most unfavorable configuration of contamination that will ensure as many contaminated differences as possible. Consider the case of time series or of a unidimensional spatial domain. For a fixed lag $h$, the set of allowable outlier constellations can be studied by specifying

$$
\begin{gathered}
\bar{Z}_{n, m}^{\zeta, h}=\left(0,0,0, \zeta_{1}, \zeta_{2}, \zeta_{3}, 0,0,0, \zeta_{4}, \zeta_{5}, \zeta_{6}, 0,0,\right. \\
\left.0, \ldots, \zeta_{m}, 0, \ldots, 0\right),
\end{gathered}
$$

i.e. constellations starting with $h$ uncontaminated locations (here $h=3$ ), followed by $h$ contaminated ones, and so on until exhaustion of the $m$ outliers. 
Consider Matheron's classical variogram estimator $\hat{\gamma}_{M}\left(h, \bar{Y}_{n}\right)=\sum_{i=1}^{n-h}\left(Y_{i+h}-\right.$ $\left.Y_{i}\right)^{2}$ (or equivalently the sample autocovariance function). Put the non-zero elements of $Z_{n, 1}^{\zeta, h}$ and $Z_{n, 2}^{\zeta, h}$ equal to $\infty$, then it is easy to see that

$$
\infty=\hat{\gamma}_{M}\left(h, \bar{Y}_{n}+\bar{Z}_{n, 1}^{\zeta, h}\right)=\hat{\gamma}_{M}\left(h, \bar{Y}_{n}+\bar{Z}_{n, 2}^{\zeta, h}\right) .
$$

Consequently, the breakdown-point is zero. Now consider a highly robust variogram estimator $\hat{\gamma}_{H R}\left(h, \bar{Y}_{n}\right)=S^{2}\left(Y_{i+h}-Y_{i}\right)$, (e.g. Genton, 1998a), where $S^{2}$ is a highly robust estimator or the variance of the process $Y_{i+h}-Y_{i}$. Typically, $S^{2}$ has breakdown-point $\lfloor(n-h) / 2-1\rfloor /(n-h)$, where $\lfloor\cdot\rfloor$ denotes the integer part. Put the non-zero elements of $Z_{n, m}^{\zeta, h}$ and $Z_{n, m+1}^{\zeta, h}$ equal to $\infty$, then we have to find the smallest value of $m$ such that

$$
\infty=\hat{\gamma}_{H R}\left(h, \bar{Y}_{n}+\bar{Z}_{n, m}^{\zeta, h}\right)=\hat{\gamma}_{H R}\left(h, \bar{Y}_{n}+\bar{Z}_{n, m+1}^{\zeta, h}\right) .
$$

This has been shown to depend on the relation between $n, m$, and $h$, and the solution is plotted in Genton (1998b, Figure 4). Note that unlike the $\mathrm{AR}(1)$ case, breakdown to infinity is the only relevant breakdown region here. Similar conclusions are valid for the sample autocovariance function, see Ma and Genton (2000).

Finally, it is worth mentioning the paper by Boente et al. (1987) on qualitative robustness for dependent observations. They consider the continuity of estimators under outlier contamination in an asymptotic context. In particular, discontinuity of the estimator is regarded as a signal of the estimator not being qualitatively robust. The concept of continuity is, however, difficult to implement in finite samples.

\section{Some Illustrative Examples}

Consider the location estimation problem

$$
Y_{i}=\mu+e_{i}
$$

with the $e_{i}$ being i.i.d. The classical definition of breakdown requires that the estimator of $\mu, \hat{\mu}$ say, be pushed towards $\pm \infty$. For example, if $\hat{\mu}$ is the mean, it is well known that one outlier suffices to achieve the divergence of $\hat{\mu}$. For the median, the classical breakdown point equals $\lfloor(n-1) / 2\rfloor / n$. As the fitted value of $Y_{i}$ equals $\hat{\mu}$, the definition of Stromberg and Ruppert gives identical results. To implement our definition, introduce the set of allowable outlier constellations such that every $\bar{Z}_{n, m}^{\zeta} \in \mathcal{Z}_{n, m}^{\zeta}$ has $n-m$ zeros, and $m$ 
non-zero elements. Put the non-zero elements of $\bar{Z}_{n, 1}^{\zeta}$ and $\bar{Z}_{n, 2}^{\zeta}$ equal to $\infty$, then it is easy to see for the mean that

$$
\infty=\hat{\mu}\left(\bar{Y}_{n}+\bar{Z}_{n, 1}^{\zeta}\right)=\hat{\mu}\left(\bar{Y}_{n}+\bar{Z}_{n, 2}^{\zeta}\right)
$$

Consequently, we obtain the same breakdown-point as Donoho-Huber or Stromberg-Ruppert. For the median, we set the non-zero elements of $\bar{Z}_{n, m}^{\zeta}$ for $m=\lfloor(n+1) / 2\rfloor$ and $m=\lfloor(n+1) / 2\rfloor+1$ to $\infty$ and obtain a similar result.

As a second example, consider the $\mathrm{AR}(1)$ model introduced earlier with one additive outlier. Define $\bar{Y}_{n}^{-}$and $\bar{Y}_{n}^{+}$as containing the first and last $n-1$ elements of $\bar{Y}_{n}$, respectively. For OLS, we obtain

$$
\hat{\phi}=\frac{\left(\bar{Y}_{n}^{+}+\bar{Z}_{n, m}^{\zeta,+}\right)^{\prime}\left(\bar{Y}_{n}^{-}+\bar{Z}_{n, m}^{\zeta,-}\right)}{\left(\bar{Y}_{n}^{-}+\bar{Z}_{n, m}^{\zeta,-}\right)^{\prime}\left(\bar{Y}_{n}^{-}+\bar{Z}_{n, m}^{\zeta,-}\right)} .
$$

Set the non-zero elements of $\bar{Z}_{n, 1}^{\zeta}$ and $\bar{Z}_{n, 2}^{\zeta}$ equal to $\zeta$. Analogous to the expression in the introduction, it is easy to show that the denominator is quadratic in $\zeta$, whereas the numerator is (at most) linear in $\zeta$. Letting $\zeta$ tend to $\infty$, we obtain $\hat{\phi} \rightarrow 0$ for both $m=1$ and $m=2$, indicating breakdown. Note, however, that the supremum bias is not reached. The supremum bias is obtained either by taking $\bar{Z}_{n, n}^{\zeta}=(\zeta, \ldots, \zeta)$ or $\bar{Z}_{n, n}^{\zeta}=(\zeta,-\zeta, \zeta,-\zeta, \ldots)$ and letting $\zeta$ tend to infinity.

Our third example concerns the non-linear regression setting. It illustrates further crucial differences between our definition and the breakdown definition of Stromberg and Ruppert. We consider the Michaelis-Menten model

$$
Y_{i}=\frac{V X_{i}}{K+X_{i}}+e_{i}=\frac{\alpha K X_{i}}{K+X_{i}}+e_{i}
$$

where $\alpha=V / K, V, K>0$, and $X_{i}>0$. For simplicity, we assume that our region of interest for $X$ is $X \in[0,3]$. It is easy to see that the functional form of the Michaelis-Menten model is non-decreasing. The main point of Stromberg and Ruppert (1992) to discuss this model is that if outliers are such that the estimator for $K$ diverges while that for $\alpha$ remains constant, the estimator is broken in the Donoho-Huber sense. The model's fit (over the range mentioned), however, is still bounded and tends to $\hat{\alpha} X$. This leads Stromberg and Ruppert to their alternative definition of breakdown. We restrict attention to their type of outliers and show that the StrombergRuppert and our definition of breakdown do not always coincide. We consider outliers of the form $Z_{i, m}^{\zeta}=(\beta \zeta, \zeta)$ for the non-zero elements in $\bar{Z}_{n, m}^{\zeta}$, where $\beta$ is a fixed bounded constant and $Z_{i, m}^{\zeta}$ is the $i$ th element of $\bar{Z}_{n, m}^{\zeta}$. So the outliers 
lie on a specific ray from the origin. We consider the least-squares estimator and let $\zeta$ tend to infinity. Clearly, if there is one extreme outlier only, the least-squares regression curve must pass through this outlier, implying

$$
\begin{aligned}
Y_{j}+\beta \zeta & =\frac{\hat{\alpha} \hat{K} \cdot\left(X_{j}+\zeta\right)}{\hat{K}+X_{j}+\zeta} \Leftrightarrow \\
\hat{\alpha} & =\frac{\left(Y_{j}+\beta \zeta\right)\left(\hat{K}+X_{j}+\zeta\right)}{\left(X_{j}+\zeta\right) \cdot \hat{K}},
\end{aligned}
$$

for the outlier pair $\left(Y_{j}, X_{j}\right)$. Using this, the least-squares objective function becomes

$$
\min _{\hat{K}} \sum_{i \neq j}\left[Y_{i}-\frac{\left(Y_{j}+\beta \zeta\right)\left(\hat{K}+X_{j}+\zeta\right) \hat{K} X_{i}}{\left(X_{j}+\zeta\right) \cdot \hat{K} \cdot\left(\hat{K}+X_{i}\right)}\right]^{2} .
$$

As the numerator of the second term within brackets is of order $\zeta^{2}$, whereas the denominator is of order $\zeta$, it is clear that the optimal $\hat{K}$ satisfies $\hat{K}=$ $\rho \zeta^{\kappa}+o_{p}\left(\zeta^{\kappa}\right)$ for some $\rho>0$ and $\kappa \geq 1$ when $\zeta \rightarrow \infty$. If $\kappa>1, \hat{\alpha}=\beta+O\left(\zeta^{-1}\right)$. Similar derivations can be followed if there are two outliers, i.e., $Z_{i, m}^{\zeta}=$ $\left(\beta \zeta_{1}, \zeta_{1}\right)$ for $i=j_{1}$, and $Z_{i, m}^{\zeta}=\left(\beta \zeta_{2}, \zeta_{2}\right)$ for $i=j_{2}$. By letting $\zeta_{1}, \zeta_{2} \rightarrow \infty$ and $\zeta_{1} / \zeta_{2} \rightarrow c$ with $1>c \geq 0$, one easily derives that the Michaelis-Menten curve collapses to a straight line through the origin with slope coefficient $\beta$, i.e., $\hat{\alpha}=\beta$ and $\hat{K} / \max \left(\zeta_{1}, \zeta_{2}\right) \rightarrow 0$. This also holds if there are more than two outliers of the type above. Note that in all these cases the fit at any $X \in[0,3]$ is bounded, such that the least-squares estimator has not broken in the sense of Stromberg and Ruppert. To answer the question whether the estimator has broken in the sense of our new definition of breakdown, we also have to consider the situation $\zeta_{1}=\zeta_{2}$. We assume that $Z_{i, m}^{\zeta}=(\beta \zeta, \zeta)$ for $m$ distinct entries of $\bar{Z}_{n, m}^{\zeta}$, and zero otherwise. Assuming the last $m$ observations are contaminated, the least-squares objective function becomes

$$
\begin{gathered}
\min _{\rho, Y^{*}, X^{*}}\left\{\sum_{i=1}^{n-m}\left[Y_{i}-\frac{X_{i}\left(Y^{*}+\beta \zeta\right)\left(X^{*}+(1+\rho) \zeta\right)}{\left(X^{*}+\zeta\right)\left(X_{i}+\rho \zeta\right)}\right]^{2}+\right. \\
\left.\sum_{i=n-m+1}^{n}\left[Y_{i}+\beta \zeta-\frac{\left(X_{i}+\zeta\right)\left(Y^{*}+\beta \zeta\right)\left(X^{*}+(1+\rho) \zeta\right)}{\left(X^{*}+\zeta\right)\left(X_{i}+(1+\rho) \zeta\right)}\right]^{2}\right\}= \\
\min _{\rho, Y^{*}, X^{*}}\left\{\sum_{i=1}^{n-m}\left[Y_{i}-\frac{\beta(1+\rho) X_{i}}{\rho}+O\left(\zeta^{-1}\right)\right]^{2}+\right. \\
\left.\sum_{i=n-m+1}^{n}\left[Y_{i}-Y^{*}-\frac{\beta \rho\left(X_{i}-X^{*}\right)}{1+\rho}+O\left(\zeta^{-1}\right)\right]^{2}\right\},
\end{gathered}
$$


where we made use of (12) with $Y_{j}=Y^{*}$ and $X_{j}=X^{*}$, and $\hat{K}=\rho \zeta$. From (15) we have

$$
Y^{*}-\beta \rho X^{*} /(1+\rho)=m^{-1} \sum_{i=n-m+1}^{n}\left(Y_{i}-\beta \rho X_{i} /(1+\rho)\right),
$$

such that (15) simplifies to

$$
\begin{gathered}
\min _{\theta \geq 1} \sum_{i=1}^{n-m}\left[Y_{i}-\theta \tilde{X}_{i}\right]^{2}+ \\
\sum_{i=n-m+1}^{n}\left[\left(Y_{i}-M_{Y}^{m}\right)-\frac{\tilde{X}_{i}-M_{X}^{m}}{\theta}\right]^{2}+O\left(\zeta^{-2}\right),
\end{gathered}
$$

with $\theta=(1+\rho) / \rho, \tilde{X}_{i}=\beta X_{i}, M_{Y}^{m}=\sum_{i=n-m+1}^{n} Y_{i} / m$, and $M_{X}^{m}=\sum_{i=n-m+1}^{n} \tilde{X}_{i} / m$. The restriction $\theta \geq 1$ follows from $\rho \geq 0$. An example of how (16) works is given in Figure 1. For a simulated data set, we contaminate the 3 observations most to the right by moving them in parallel to the ray $\beta X$. Using (16), we are looking for a $\theta$ (or $\theta \beta$ ) such that the squared vertical discrepancies between the observations and the pictured line segments are minimal. Note that the contaminated observations (solid) are taken in deviation of the right-hand line segment, whereas the uncontaminated (open) observations are taken in deviation of the left-hand line segment. If $\theta \geq 1$ is binding both line segments have the same slope, though different intercepts. The fit over the range $X \in[0,3]$ is then given by $\beta X$, which does not vary by adding additional outliers. This will be the case if for example $\beta \geq \max _{i} Y_{i} / X_{i}$. If $\beta$ is smaller, the constraint $\theta \geq 1$ may not be binding and the fit $\hat{\theta} X$ still varies because $\hat{\theta}$ still depends on the sample through (16). So with $m$ outliers, the boundary badness set for extreme outliers and given $X \in[0,3]$ is given by either $\left\{\beta X, \hat{\theta}_{m} X\right\}$ or $\{\beta X\}$, where $\hat{\theta}_{m}$ can still vary for increasing $m$. It is clear, however, that the intersection of these sets for $m=1$ and $m=2$ is non-empty. Therefore, the breakdown-point of the least-squares estimator is 0 . The model fit, however, is still finite in all cases considered. Therefore, the estimator may have broken down in the sense of Section 2 without having been broken in the sense of Stromberg and Ruppert.

Note that the use of the boundary set of possible badness outcomes also provides additional information. In particular, there are two types of outlier configurations of interest. If outliers lie increasingly far apart, the model's fit is dictated completely by the outliers $(\beta X)$. The uncontaminated observations have no influence on the estimator any more. This leads to our notion of the estimator having broken down, even if the model's fit is below 


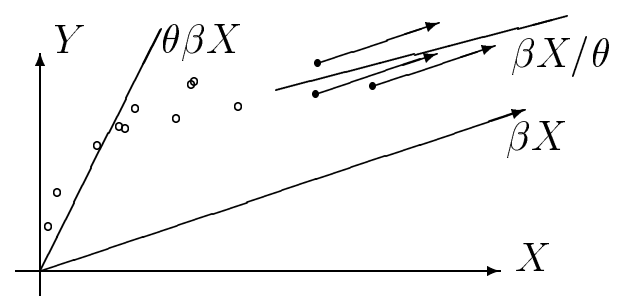

Figure 1: LS fit of the Michaelis-Menten model with three outliers

its supremum. If however, $\beta>0$ is sufficiently small and the outliers occur in one cluster, the model's fit still bears some relation to the true fit as $\hat{\theta}_{m}$ still depends on the uncontaminated sample observations. So the estimator has not broken down according to our definition if only outliers in clusters $\left(\zeta_{i} \equiv \zeta\right)$ are considered.

\section{Asymptotic Breakdown-Point}

To extend our definition to the asymptotic case, we introduce the stochastic processes $\bar{Y}=\left\{Y_{i}\right\}_{i=1}^{\infty}$ and $\bar{Z}_{\pi}^{\zeta}=\left\{Z_{i}^{\zeta}\right\}_{i=1}^{\infty}$. The interpretation is similar to that in Section 2. The subscript $\pi$ for the contaminating process indicates the fraction or probability of contamination. For example, for regression outliers $\bar{Z}_{\pi}^{\zeta}$ typically is an i.i.d. process with $P\left(Z_{i}^{\zeta}=\zeta\right)=1-P\left(Z_{i}^{\zeta}=0\right)=\pi$ for all $n$. The badness measure is now a functional,

$$
R\left(\theta, \bar{Y}+\bar{Z}_{\pi}^{\zeta}\right)
$$

We also examine the boundary badness set

$$
\bar{R}\left(\bar{Y}, \mathcal{Z}_{\pi}^{\zeta}\right)=\partial\left(\bigcup_{\bar{Z}_{\pi}^{\zeta} \in \mathcal{Z}_{\pi}^{\zeta}} R\left(\hat{\theta}\left(\bar{Y}+\bar{Z}_{\pi}^{\zeta}\right), \bar{Y}\right)\right)
$$

where $\overline{\mathcal{Z}}_{\pi}^{\zeta}$ contains the allowable contaminating processes corresponding to a contaminating probability $\pi$.

Definition 2 The breakdown-point $\varepsilon\left(\hat{\theta}, \bar{Y}, \overline{\mathcal{Z}}_{\pi}^{\zeta}\right)$ of the estimator $\hat{\theta}$ at the (uncontaminated) process $\bar{Y}$ for the set of allowable outlier configurations $\overline{\mathcal{Z}}_{\pi}^{\zeta}$, is 
given by

$$
\begin{gathered}
\varepsilon\left(\hat{\theta}, \bar{Y}, \overline{\mathcal{Z}}_{\pi}^{\zeta}\right)=\inf \left\{\pi \mid \exists \bar{\delta}>0: \lim _{\zeta \rightarrow \infty} \bar{R}\left(\bar{Y}, \mathcal{Z}_{\pi}^{\zeta}\right) \bigcap\right. \\
\left.\lim _{\zeta \rightarrow \infty} \bar{R}\left(\bar{Y}, \mathcal{Z}_{\pi+\delta}^{\zeta}\right) \neq \emptyset \quad \forall 0<\delta<\bar{\delta}\right\} .
\end{gathered}
$$

If the probability measure of $\bar{Y}$ has atoms, the condition inside the definition of the breakdown-point should hold for all slightly perturbed processes $\bar{Y}$, where the extent of perturbation tends to zero. The present definition requires the boundary of the set of possible badness values to be flat in at least one direction over a non-degenerate region $[\pi, \pi+\bar{\delta}]$.

To illustrate the definition, consider the mean as a location estimator in the i.i.d. setting,

$$
\hat{\theta}(\bar{Y})=\int Y_{i} P\left(d Y_{i}\right)
$$

As a badness measure, consider the bias $|\hat{\theta}-\theta|$. This should give us the Donoho-Huber breakdown-point of the mean. For the i.i.d. contaminating process $P\left(Z_{i}^{\zeta}=\zeta\right)=1-P\left(Z_{i}^{\zeta}=0\right)=\pi$ with $\zeta \uparrow \infty$, it is easy to show that for every positive $\pi$ arbitrarily close to 0 , both $+\infty$ and $-\infty$ are contained in the boundary badness set. Consequently, the breakdown-point of the mean is zero. A similar line of argument can be used to show that the median has a breakdown-point of $1 / 2$.

We conclude with a more involved example: the LMS estimator for timeseries models, see Rousseeuw and Leroy (1987), and Lucas (1997). Consider the $\operatorname{AR}(1)$ model

$$
Y_{i}=\phi Y_{i-1}+e_{i}
$$

where the innovations $e_{i}$ form an i.i.d. process. We assume that $e_{i}$ is symmetrically distributed around 0 and that badness is measured by bias.

Consider the two i.i.d. additive outlier processes

1. $P\left(Z_{i}^{\zeta}=\zeta\right)=1-P\left(Z_{i}^{\zeta}=0\right)=\pi$

2. $P\left(Z_{i}^{\zeta}=\zeta\right)=P\left(Z_{i}^{\zeta}=\gamma \zeta\right)=\left(1-P\left(Z_{i}^{\zeta}=0\right)\right) / 2=\pi / 2$;

where $\gamma \in[-1,1]$ is a fixed constant. The LMS estimator $\hat{\theta}_{L M S}$ of $\phi$ minimizes

$$
\operatorname{median}\left(e_{i}+Z_{i}^{\zeta}-\hat{\theta}_{L M S} Z_{i-1}^{\zeta}+\left(\phi-\hat{\theta}_{L M S}\right) Y_{i-1}\right)^{2},
$$


which means that it solves

$$
\begin{aligned}
0.5= & (1-\pi)^{2} P\left(\left[e_{i}+\left(\phi-\hat{\theta}_{L M S}\right) Y_{i-1}\right]^{2}<c\right)+ \\
& \pi(1-\pi) P\left(\left[e_{i}+\left(\phi-\hat{\theta}_{L M S}\right) Y_{i-1}+\zeta\right]^{2}<c\right)+ \\
& \pi(1-\pi) P\left(\left[e_{i}+\left(\phi-\hat{\theta}_{L M S}\right) Y_{i-1}-\hat{\theta}_{L M S} \zeta\right]^{2}<c\right)+ \\
& \pi^{2} P\left(\left[e_{i}+\left(\phi-\hat{\theta}_{L M S}\right) Y_{i-1}+\left(1-\hat{\theta}_{L M S}\right) \zeta\right]^{2}<c\right)
\end{aligned}
$$

with respect to $c$ for contaminating process 1 , and

$$
\begin{aligned}
0.5= & (1-\pi)^{2} P\left(\left[e_{i}+\left(\phi-\hat{\theta}_{L M S}\right) Y_{i-1}\right]^{2}<c\right)+ \\
& \pi(1-\pi) P\left(\left[e_{i}+\left(\phi-\hat{\theta}_{L M S}\right) Y_{i-1}+\zeta\right]^{2}<c\right) / 2+ \\
& \pi(1-\pi) P\left(\left[e_{i}+\left(\phi-\hat{\theta}_{L M S}\right) Y_{i-1}+\gamma \zeta\right]^{2}<c\right) / 2+ \\
& \pi(1-\pi) P\left(\left[e_{i}+\left(\phi-\hat{\theta}_{L M S}\right) Y_{i-1}-\gamma \hat{\theta}_{L M S} \zeta\right]^{2}<c\right) / 2+ \\
& \pi(1-\pi) P\left(\left[e_{i}+\left(\phi-\hat{\theta}_{L M S}\right) Y_{i-1}-\gamma \hat{\theta}_{L M S} \zeta\right]^{2}<c\right) / 2+ \\
& \pi^{2} P\left(\left[e_{i}+\left(\phi-\hat{\theta}_{L M S}\right) Y_{i-1}+\left(1-\hat{\theta}_{L M S}\right) \zeta\right]^{2}<c\right) / 4 \\
& \pi^{2} P\left(\left[e_{i}+\left(\phi-\hat{\theta}_{L M S}\right) Y_{i-1}+\left(\gamma-\hat{\theta}_{L M S}\right) \zeta\right]^{2}<c\right) / 4 \\
& \pi^{2} P\left(\left[e_{i}+\left(\phi-\hat{\theta}_{L M S}\right) Y_{i-1}+\left(1-\gamma \hat{\theta}_{L M S}\right) \zeta\right]^{2}<c\right) / 4 \\
& \pi^{2} P\left(\left[e_{i}+\left(\phi-\hat{\theta}_{L M S}\right) Y_{i-1}+\gamma\left(1-\hat{\theta}_{L M S}\right) \zeta\right]^{2}<c\right) / 4
\end{aligned}
$$

for process 2 . If we let $\zeta \rightarrow \infty$, we see that there are only 3 and 4 interesting values of $\hat{\theta}_{L M S}$ for contaminating processes 1 and 2, respectively. For process 1 , we only consider $0, \phi$, and 1 , while for process 2 we also consider $\hat{\theta}_{L M S}=\gamma$. We can now rewrite $(22)$ as

$$
c= \begin{cases}P_{(e+\phi Y)^{2}}^{-1}\left(0.5(1-\pi)^{-1}\right) & \text { for } \hat{\theta}_{L M S}=0, \\ P_{e^{2}}^{-1}\left(0.5(1-\pi)^{-2}\right) & \text { for } \hat{\theta}_{L M S}=\phi, \\ P_{(e+(\phi-1) Y)^{2}}^{-1}\left(0.5\left(1-2 \pi+2 \pi^{2}\right)^{-1}\right) & \text { for } \hat{\theta}_{L M S}=1 .\end{cases}
$$

where $P_{X}^{-1}(\cdot)$ is the inverse c.d.f. corresponding to the random variable $X$. It is clear that for $\pi$ sufficiently small, the second branch dominates. For $\phi$ near 1 and $\pi$ sufficiently large, however, the third branch dominates. Finally, for $\phi$ sufficiently far from unity and $\pi$ sufficiently large, the first branch dominates. A similar derivation can be set up for the second contaminating process, revealing that for $\phi$ and $\gamma$ sufficiently close and $\pi$ sufficiently large, setting $\hat{\theta}_{L M S}=\gamma$ gives the LMS estimator. If $\pi$ increases further, however, setting $\hat{\theta}_{L M S}$ either to $0,+1$, or -1 gives a lower median of squares. Two examples of the objective functions of the LMS estimator for the Gaussian $\operatorname{AR}(1)$ are given in Figure 2. Note that $\gamma=-1$ has to be treated differently from $|\gamma|<1$, because an additional term is non-negligible in the expression 
$\varphi=0.50 ; \gamma=0.52$

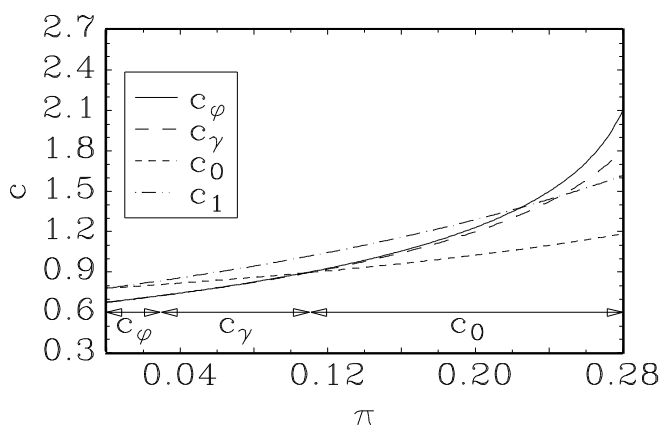

$\varphi=0.95 ; \gamma=0.97$

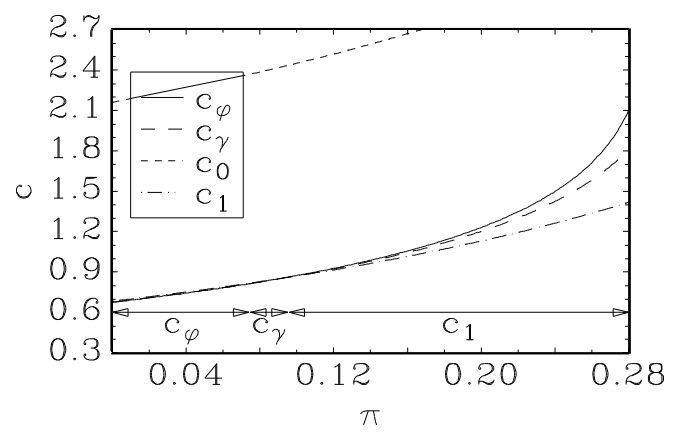

Figure 2: Median of squares for the Gaussian AR(1) for the type 1 contamination and $\hat{\theta}_{L M S}=\phi\left(c_{\phi}\right)$, the type 2 contamination and $\hat{\theta}_{L M S}=\gamma\left(c_{\gamma}\right)$, the type 1 contamination and $\hat{\theta}_{L M S}=0\left(c_{0}\right)$, and the type 1 contamination and $\hat{\theta}_{L M S}=1\left(c_{1}\right)$, where $\pi$ denotes the fraction of contamination. At the bottom of each graph the arrows indicate the range over which each of the objective values dominates.

for $c$. As $\gamma$ was left unspecified, we obtain that the set of possible badness values is expanding up to the value of $\pi$ for which (breakdown to zero)

$$
\begin{gathered}
P_{(e+\phi Y)^{2}}^{-1}\left([2-2 \pi]^{-1}\right) \leq \\
P_{(e+(\phi-\gamma) Y)^{2}}^{-1}\left(\left[2-4 \pi+5 \pi^{2} / 2\right]^{-1}\right),
\end{gathered}
$$

or (breakdown to +1$)$

$$
\begin{aligned}
& P_{(e+(\phi-1) Y)^{2}}^{-1}\left(\left[2-4 \pi+4 \pi^{2}\right]^{-1}\right) \leq \\
& P_{(e+(\phi-\gamma) Y)^{2}}^{-1}\left(\left[2-4 \pi+5 \pi^{2} / 2\right]^{-1}\right),
\end{aligned}
$$

or (breakdown to -1$)$

$$
\begin{aligned}
& P_{(e+(\phi+1) Y)^{2}}^{-1}\left(\left[2-4 \pi+3 \pi^{2}\right]^{-1}\right) \leq \\
& P_{(e+(\phi-\gamma) Y)^{2}}^{-1}\left(\left[2-4 \pi+5 \pi^{2} / 2\right]^{-1}\right)
\end{aligned}
$$

for all $\gamma \in[-1,1]$. For larger values of $\pi$, the boundary badness set is nonexpanding and the maximum bias curve is flat over some part of the support and equal to either $\phi, 1-\phi$, or $-1-\phi$. Note that this may not coincide with the maximum bias, which is attained by letting $\pi$ increase further. If we set $\pi$ close to 0.5 , we obtain the LMS estimate of $\hat{\theta}_{L M S}=1$. Before $\pi=0.5$, however, the LMS estimator may already have been broken to either 0 or -1 . Also note that this form of breakdown does not correspond to Genton's 
definition, where the objective function has to diverge. As shown above, the LMS may still be finite while the estimator has broken to 0 or 1 .

A picture of the breakdown-curve of the LMS estimator in the Gaussian $\mathrm{AR}(1)$ is given in Figure 3. Clearly, the breakdown-point varies with the true probability measure of the stochastic process, i.e., with the value of $\phi$. The breakdown-point is very close to zero for $\phi$ near $-1,0$, and 1 . Moreover, there are two kinks in the curve. The left kink reveals the point where breakdown towards -1 is superseded by breakdown towards 0. Similarly, the second kink gives the point where breakdown to 1 dominates breakdown to 0 . Using our definition of breakdown, it is clear that the breakdown-point of the (highly robust) LMS estimator in a time-series context is far below 0.5, and even far below $0.5 /(p+1)$ with $p$ the order of the autoregression.

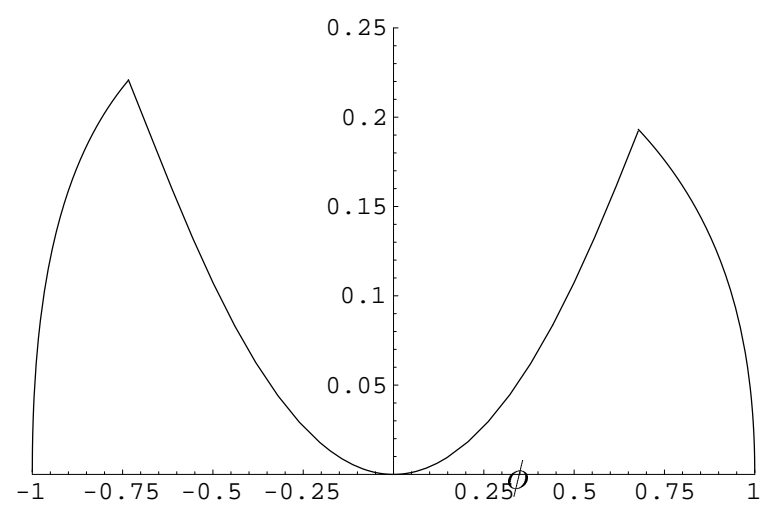

Figure 3: Breakdown-curve of the LMS estimator in the Gaussian AR(1) as a function of $\phi$.

\section{Concluding Remarks}

We introduced a new concept of breakdown, applicable to settings with independent and dependent observations. Using examples from linear and non-linear regression, time-series, and spatial statistics, we showed that our new definition comprises most of the familiar notions of breakdown. In some cases, however, our definition differs from the traditional ones. Of particular interest is the setting of simple autoregressive time series, where our breakdown-point illustrates the estimator has been broken, whereas traditional definitions still do not indicate breakdown. A completely different example from the non-linear regression setting revealed similar patterns. 


\section{References}

[1] Boente, G., Fraiman, R., and Yohai, V. J. (1987), "Qualitative Robustness for Stochastic Processes," The Annals of Statistics, 15, 1293-1312.

[2] Donoho, D. L., and Huber, P. J. (1983), "The Notion of Breakdown Point," in A Festschrift for Erich L. Lehmann, eds. P. J. Bickel, K. A. Doksum, and J. L. Hodges, Jr. Belmont, CA: Wadsworth, pp. 157-184.

[3] Genton, M. G. (1998a), "Highly Robust Variogram Estimation," Mathematical Geology, 30, 213-221.

[4] Genton, M. G. (1998b), "Spatial Breakdown Point of Variogram Estimators," Mathematical Geology, 30, 853-871.

[5] Hampel, F. R. (1971), "A General Qualitative Definition of Robustness," The Annals of Mathematical Statistics, 42, 1887-1896

[6] He, X. (1991), “A Local Breakdown Property of Robust Tests in Linear Regression," Journal of Multivariate Analysis, 38,294-305.

[7] He, X., and Simpson, D. G. (1992), "Robust Direction Estimation,", The Annals of Statistics, 20, 351-369.

[8] He, X., and Simpson, D. G. (1993), "Lower Bounds for Contamination Bias: Globally Minimax Versus Locally Linear Estimation," The Annals of Statistics, 21,314-337.

[9] He, X., Simpson, D. G., and Portnoy, S. L. (1990), "Breakdown Robustness of Tests," Journal of the American Statistical Association, 85, 446-452.

[10] Lucas, A. (1997), "Asymptotic Robustness of Least Median of Squares for Autoregressions with Additive Outliers," Communications in Statistics-Theory and Methods, 26, 2363-2380.

[11] Ma, Y., and Genton, M. G. (2000), "Highly Robust Estimation of the Autocovariance Function," Journal of Time Series Analysis, to appear.

[12] Martin, R. D. (1980), "Robust Estimation of Autoregressive Models," in Directions in time series (Proc. Meeting, Iowa State Univ., Ames, Iowa, 1978), Hayward, CA: Inst. Math. Statist., pp. 228-262. 
[13] Martin, R.D., and J. De Jong (1977), "Asymptotic properties of robust generalized M-estimates for the first-order autoregressive parameter," Bell Laboratories Technical Memo, Murray Hill, New Jersey.

[14] Rousseeuw, P. J., and Leroy, A. M. (1987), Robust Regression \& Outlier Detection, New York: Wiley.

[15] Sakata, S., and White, H. (1995), "An Alternative Definition of Finite Sample Breakdown Point with Applications to Regression Model Estimators," Journal of the American Statistical Association, 90, 1099-1106.

[16] Sakata, S., and White, H. (1998), "High Breakdown Point Conditional Dispersion Estimation with Application to S\&P 500 Daily Returns Volatility," Econometrica, 66, 529-567.

[17] Stromberg, A. J., and Ruppert, D. (1992), "Breakdown in Nonlinear Regression," Journal of the American Statistical Association, 87, 991997. 